\title{
Connecting neuronal circuits for movement
}

\author{
Silvia Arber ${ }^{1,2}$ and Rui M. Costa ${ }^{3,4}$ \\ ${ }^{1}$ Biozentrum, University of Basel, 4056 Basel, Switzerland. \\ ${ }^{2}$ Friedrich Miescher Institute for Biomedical Research, 4058 Basel, Switzerland. \\ ${ }^{3}$ Zuckerman Mind Brain Behavior Institute, Columbia University, New York, NY 10027, USA. \\ ${ }^{4}$ Champalimaud Research, Champalimaud Centre for the Unknown, Lisbon 1400-038, Portugal.
}

Corresponding authors:

silvia.arber@unibas.ch; rc3031@,columbia.edu 
Movement is the most common final output of nervous system activity and is essential for survival. But what makes this seemingly trivial statement so scientifically challenging? Neurons that contribute to when and how our body moves are distributed throughout the nervous system. Thus, even a simple movement such as arm flexion requires the coordinated activation of many different neuronal populations across multiple brain regions. A key question is how the nervous system produces diverse and precise actions aligned with the organisms' behavioral needs. These processes are affected in diseases such as Parkinson's or Huntington's, in which aberrant motor behavior dominates. Recent studies are transformative in how we think about the control of movement. A common denominator of these studies is that brain regions that contribute to motor behavior can no longer be considered as interacting boxes. Instead, deep circuit-level insight based on specific neuronal populations emerges as being critical to revealing motor system organization and understanding its function. It is likely that insights at this level can also help to design more specific and direct interventions for diseases of the motor system and neuroprosthetics applied after injuries.

Executive circuits eliciting body movements reside in the spinal cord. Although one might think of these circuits simply as the engine for movement, a striking complexity and organizational logic of spinal circuit architecture is being unraveled. Large numbers of transcriptionally and likely also functionally distinct neurons are generated during development (1). Moreover, in contrast to primary sensory systems such as olfaction, whether an organizational logic exists at the output steps within the motor system was unclear. We now know that circuits regulating the functionally opposing extensor and flexor muscles are connected into distinct and spatially separate spinal submodules in mice (2), and that in zebrafish, even within one genetic class, neurons can be subdivided by function aligned to different swimming speeds (3). Although much work lies ahead, one can speculate that specialized spinal microcircuits and their associated sensory feedback loops are the recipients of motor commands from the brain, and that these microcircuits are essential substrates to produce diverse actions as the behavioral output of the brain. 
The spinal cord alone cannot generate sustained movement. Best support for this statement comes from patients with complete spinal cord injury in whom body parts controlled by spinal segments below the site of injury are permanently paralyzed despite functional local spinal circuits. This observation raises the important question of the origin of motor commands that provide instructions for body movement to spinal circuits. The brainstem is a major source of synaptic input to the spinal cord. Diverse motor-related neuronal activity patterns are observed there, but historically, it has been difficult to assign functions to these neurons because of the lack of cell type identification. However, studies that apply genetic and circuit connectivity approaches to the brainstem in multiple species are beginning to unravel stunning organizational patterns. In the fruit fly, Drosophila melanogaster, a large screen that assesses by means of machine vision the impact of genetically distinct neuronal populations on behavior provides a starting point for further dissections of neuron-to-action maps (4). Studies of the mouse brainstem have also provided headway. On the basis of behavioral differences between fore- and hindlimbs, experiments to probe connectivity patterns of brainstem neurons to spinal motor neurons that target these two extremities revealed striking distinctions (5). A defined glutamatergic population of neurons within the medulla is dedicated specifically to the grasping phase of forelimb usage without implication in the full-body behavior locomotion (5). Another distinct neuronal population within the brainstem's lateral paragigantocellular nucleus is required for high-speed locomotion (6). Using developmental transcription factor expression to stratify neuron function, a specific descending glutamatergic brainstem population was implicated in halting locomotion (7). As a last, specialized example, a glutamatergic population marked by expression of corticotropin-releasing hormone controls urine release by regulating bladder contraction (8).

These exemplary studies convey two main messages. First, brainstem areas use division of labor to control diversification of body movement. Executed behaviors range from full-body forms, including locomotion and orientation, to skilled movement sequences of body parts during forelimb manipulation behaviors. Second, within a brainstem region, functionally diverse neurons are frequently intermingled. This is the likely reason why progress in assigning functions to neurons was slow, and only application of additional 
criteria-including projection targets, neurotransmitter identity, or genetic identity-provided breakthroughs. Conceptually, we consider brainstem neurons defined by these criteria as command lines for action. For these, a controlled action element can include a longer specific behavior (for example, locomotion), a behavioral syllable (for example, arm flexion), or a parameter important for behavior (for example, locomotor speed). These findings have potential for understanding the dysfunctional nervous system - for example, by clarifying walking circuits at the brainstem level that could be specifically manipulated to shortcut higher basal ganglia dysfunctions in Parkinson's disease.

A corollary of these ideas is that brain centers without direct access to spinal executive centers must relate to and be integrated with this command line repertoire to influence body movements. But how does this work? An organism has to choose which action is appropriate in a particular context and decide when to do it and how vigorously. Such computations are mostly performed upstream of brainstem command lines, in brain circuits, including the cortex, basal ganglia, hypothalamus, and superior colliculus, the combined outputs of which determine when and which command lines are active and how vigorously. Moreover, the cerebellum, a brainstem-associated structure, rapidly predicts the sensory consequences of movement and allows animals to adjust movement. It can also coordinate movements between different body parts and permits feedforward control of specific movements. How the combined activity of upstream circuits leads to the activation of specific command lines is not a trivial problem to address - especially considering that each command line has different inputs regulating it, and that most upstream circuits involved in choice and coordination also connect to more than one command line.

It is easier to comprehend action choice when it is driven by strong external stimuli than by cognitive decision-making processes guiding voluntary behaviors. A classic example of the first category is the innate behavioral interaction between prey and predator. Two opposing motor programs - predation or evasionare regulated by distinct intermingled neuronal subpopulations within the hypothalamus (9). Additionally, the superior colliculus is an important hub to compute inputs from many brain areas, including sensory 
modalities, and mediate choice, as exemplified for different forms of defensive behavior in mice in response to looming stimuli mediated by subpopulations of Parvalbumin-expressing neurons (10). In both casesresponses to predator or prey and the choice to engage in defensive behavior-neurons are wired into distinct circuits that indirectly communicate with differential downstream command circuits in the brainstem and spinal cord to elicit actions.

Understanding action choice in cognitive and voluntary tasks is much more challenging. The basal ganglia are brain areas that receive excitatory inputs from the cortex and the thalamus, as well as dopaminergic modulation from the midbrain; the output of these areas can modulate brainstem circuits. The basal ganglia are therefore in an ideal position to influence which command lines should be activated under particular circumstances, and how vigorously (11). Accordingly, activity in the striatum, the main basal ganglia input structure, is action specific (12). Whereas information about which particular action to execute seems to arrive at the basal ganglia from the cortex (or from the thalamus), basal ganglia output to brainstem circuits conveys the commitment to execute that action (13). This commitment and its vigor are modulated by dopaminergic inputs to basal ganglia, which do not exhibit action-type specificity (14). Therefore, diverse structures provide information to the basal ganglia, which govern the commitment and vigor to act. How basal ganglia output structures precisely intersect with command lines and executive circuits requires further study.

The motor cortex is the evolutionarily most recent structure of the motor system but also the most controversial one to which to assign behavioral functions. Early studies demonstrate that cats without a motor cortex can still perform a large behavioral repertoire, supporting a model in which subcortical structures play dominant roles in movement control. An intriguing feature of cortical neurons is that they are the only class of supraspinal neurons that directly communicate with most other motor structures, including the basal ganglia, thalamus, midbrain, brainstem, and spinal circuits. Through this broadcasting mechanism, they can convey and distribute context-dependent and cognitive information widely. 
Conceptually, motor cortex output can both serve as a command line and influence action choice via the modulation of other command lines. A growing body of evidence suggests that cortical areas endowed with movement regulation might do so preferentially in settings in which context dependence or cognitive control mechanisms are involved in deciding action programs $(13,15)$. An important step to clarify motor cortex function will be to determine how specific neuronal populations target and functionally affect subcortical circuits in different species, hopefully clarifying why humans but not evolutionarily lower species can execute exquisite skilled movements, such as playing the violin.

Major open questions in the field remain: How do brain regions and their neuronal subpopulations interact with each other to coordinate movement? How does action choice and repression occur, especially when cognition is involved? How is motor learning implemented at the circuit level, and what are the temporal and spatial dynamics of this process? Some of these questions will likely require new computational frameworks that deal with the dynamics of complex interacting systems. But the field of motor system research is ready for this challenge. 
References

1. J. B. Bikoff et al., Cell 165, 207 (2016)

2. M. Tripodi et al., Nature 479, 61 (2011)

3. K. Ampatzis et al., Neuron 83, 934 (2014)

4. A. A. Robie et al., Cell 170, 393 (2017)

5. M. S. Esposito et al., Nature 508, 351 (2014)

6. P. Capelli et al., Nature 551, 373 (2017)

7. J. Bouvier et al., Cell 163, 1191 (2015)

8. X. H. Hou et al., Cell 167, 73 (2016)

9. Y. Li et al., Neuron 97, 911 (2018)

10. C. Shang et al., Nat Comm. 9, 1232 (2018)

11. E. A. Yttri, J. T. Dudman, Nature 533, 402 (2016)

12. A. Klaus et al., Neuron 96, 949 (2017)

13. D. Thura, P. Cisek, Neuron 95, 1160 (2017)

14. J. A. da Silva et al., Nature 554, 244 (2018)

15. K. Svoboda, N. Li, Curr. Opin. Neurobiol. 49, 33 (2017) 